ISSN 0001-6002/2010/52/4/203-210 Acta Médica Costarricense, (C2010 Colegio de Médicos y Cirujanos

\title{
Aspectos médico legales del síndrome del menor agredido
}

\author{
(Legal-Medical Aspects of the Battered Child Syndrome)
}

\author{
Édgar Alonso Madrigal-Ramírez
}

\section{Resumen}

El Síndrome del Menor Agredido en cuya génesis participan múltiples factores biopsicosociales, afecta a niños de todas las edades alrededor del mundo, principalmente a niños pequeños y con ciertos factores de riesgo. Aunque los daños físicos que produce suelen ser transitorios, pueden ocurrir secuelas físicas y generalmente psicológicas persistentes, o la muerte. En Costa Rica existe amplia legislación que ampara al menor contra los maltratos, correspondiéndole al equipo del sistema de salud nacional salvaguardar su salud y, en muchos casos, su vida. En este contexto, le compete al médico ser un ejecutante habilidoso en la valoración clínica a través de la cual se aborde al paciente. Desde el interrogatorio no revictimizante, el personal de salud debe realizar un abordaje inmediato conducente a evitar mayor lesionología para con el niño del que se ha abusado física y sexualmente, y para lo cual debe comprender temas tales como: establecimiento de la compatibilidad del mecanismo y fisiopatología del trauma, diagnóstico diferencial y evaluación clínico forense, además de contar con elementos que identifiquen indicadores psicológicos en la dinámica traumatogénica del menor. Especial atención debe prestarse a la toma, conservación y manipulación de las muestras biológicas en casos de delitos sexuales. con el fin de evitar la pérdida de evidencia forense.

Descriptores: Síndrome del niño agredido, síndrome del niño sacudido, traumatología forense, revictimización

\section{Abstract}

The battered child syndrome, whose genesis involves multiple biopsychosocial factors, affects children of all ages around the world, especially young children with certain risk factors. Although the physical damage that occurs could be temporary, physical scars can also occur and, particularly, psychological ones, that are usually persistent or even death. In Costa Rica there is a comprehensive legislation that protects children against abuse, corresponding to the national health system team to safeguard their health and often their lives. In this context, it is up to the Doctor to be a skilled performer in the clinical assessment through which the patient is to be addressed. From a non revictimizing interrogation, health personnel should make an immediate approach conductive to avoid further damage to the child who has been physically and sexually abused. Such personnel should understand the mechanism and pathophysiology of trauma, the differential diagnosis and the clinical forensic assessment, in addition to be able to identify psychological indicators of the origin of the child's trauma. Particular attention should be given to the seizure, storage and handling of biological samples in cases of sexual crimes in order to avoid the loss of forensic evidence.

Keywords: battered child syndrome, shaken child syndrome, forensic traumatology, revictimization

Recibido: 15 de febrero de 2010

Aceptado: 22 de junio de 2010

Correo electrónico: doctoralonso@yahoo.com
Especialista en Medicina Poder Judicial. Profesor U.C.R.

Correspondencia: 
La agresión a menores es un tema de Salud Pública de proporciones mundiales; para darse una idea del problema, en Estados Unidos 4,9 de cada 1000 niños son agredidos, con fallecimiento de uno de cada mil, ${ }^{1}$ y mayor exposición de los niños de cuatro a seis años de edad. ${ }^{2}$ En Costa Rica el número de casos evaluados en la Sección de Clínica Médico Forense del Poder Judicial, crece año con año.

La perspectiva de análisis en este tipo de casos valorará siempre al menor inmerso en un ambiente, y no como un mero ente biológico, ${ }^{2}$ sobre todo porque este síndrome es una patología que altera el equilibrio biopsicosocial del menor. Los estratos socioeconómicos más bajos son más proclives, aunque ningún otro nivel está exento. ${ }^{3,4}$

El médico cargará sobre sus hombros la valoración clínica del niño, en principio con fines terapéuticos y rehabilitatorios, sin embargo, no puede perder de vista el enfoque médico legal que debe dársele al caso, ya sea porque puede ser convocado a una audiencia judicial, o bien, para imprimir un diagnóstico que no permita que los sistemas de salud involucren falsamente a familias en un problema como el señalado. ${ }^{1,3}$

En algunas ocasiones se han observado casos con abordajes incompletos, poco sistematizados por parte de los equipos de salud a nivel nacional, principalmente a través de la revisión de los expedientes a los que el médico forense tiene acceso. Este trabajo pretende actualizar elementos médico legales básicos, que el profesional debe considerar en su práctica al tratar con un posible caso.

\section{Definición médico legal}

Desde la perspectiva médico legal, el Síndrome de Menor Agredido (SMA) se puede definir como el cuadro clínico caracterizado por los daños agudos o crónicos en la salud de un menor, como consecuencia de una serie de agresiones debidas a trauma físico, psicológico-emocional, etc., que pueden llevarlo a un estado secuelar en múltiples áreas de la vida, u ocasionarle la muerte. ${ }^{5-10}$

Esta serie de agresiones suele ser ejercida por parte de los propios padres o encargados del menor. Es frecuente que los agresores tengan una historia de maltrato durante su propia infancia, cursen con problemas de estrés, financieros, de adicciones, inexperiencia en el manejo de la paternidad, etc. Estos, cuando se ven obligados a consultar al Servicio de Emergencia, tienden a dar explicaciones contradictorias o no congruentes con respecto al cuadro lesionológico del niño. ${ }^{5-7}$ Los factores de riesgo propios del menor suelen caracterizarse por prematuridad, llanto constante o excesiva demanda de atención, entre otros. ${ }^{5}$

La violencia ejercida puede ser de tipo pasiva (por omisión de cuidados), pudiéndose dar la privación de alimentos al niño (riesgo de desnutrición), el abandono físico, las carencias afectivas, la despreocupación por la aplicación del cuadro básico de vacunación y fármacos prescritos, o la negligencia en la búsqueda de atención médica oportuna. ${ }^{1,6,7} \mathrm{La}$ forma activa es la más conocida, $\mathrm{y}$ se refiere al castigo físico, principalmente concretado con las propias manos del agresor o con algún tipo de objeto (fajas, puntapiés, cables, etc.). ${ }^{1}$

El síndrome suelen sufrirlo niños muy pequeños; se sabe que hasta un $70 \%$ de los casos suceden a menores de tres años, un 50\% a menores de un año, y un 30\% a menores de seis meses (Según Rabouille, Strauss y Mc Henry, respectivamente). ${ }^{5}$

\section{Sustrato legal costarricense}

La violencia ejercida puede acarrear consigo la génesis de lesiones de diverso tipo e intensidad, clasificándose desde el punto de vista penal en lesiones levísimas (contravención), leves, graves o gravísimas (artículos 380, 125, 124 y 123 del Código Penal), dependiendo de las incapacidades temporales y permanentes que el menor requiera; pero también se establecen penas por castigos inmoderados a los hijos (artículo 382 del Código Penal) o a quien, teniendo bajo su cuidado a un menor, lo expusiera a un peligro predecible o evitable. El artículo 142 de dicho código establece pena a quien pusiere en grave peligro la salud o la vida al abandonar a su suerte a una persona incapaz de valerse por sí misma, o que deba mantener o cuidar, al colocarla en estado de desamparo físico. ${ }^{11}$

El artículo 49 del Código de la Niñez y la Adolescencia indica que los directores y el personal encargado de los centros de salud públicos o privados, adonde se lleven personas menores de edad para atenderlas, así como también las autoridad y el personal de centros educativos, guarderías o cualquier otro sitio en donde permanezcan, les corresponderá denunciar los maltratos o abusos que se propinen a niños o adolescentes; ${ }^{12}$ esto sin perjuicio de que todo ciudadano podría convertirse en cómplice al callar ante la violencia de que pueda ser víctima un menor.

Este mismo código, en el artículo 134 reza: ... “ Comprobada en sede administrativa la existencia de indicios de maltrato o abuso en perjuicio de una persona menor de edad, la denuncia penal deberá plantearse en forma inmediata. La persona o institución que actúe en protección de los menores, no podrá ser demandada, aun en caso de que el denunciado no resulte condenado en esta sede"... En otras palabras, la persona que interponga la denuncia judicial, previa comprobación de indicios de maltrato, no podrá ser demandada independientemente del resultado del proceso.

\section{Interrogatorio médico}

El objetivo del interrogatorio médico es consignar aspectos relevantes que puedan ayudar a explicar los mecanismos fisiopatológicos de los traumatismos y hallazgos producidos. ${ }^{14,15}$ 
Todo el personal de salud deberá evitar la victimización secundaria, pues esto podría promover una actualización de los hechos sucedidos al menor y configurarse en una nueva experiencia traumática. ${ }^{13}$ Si bien el interrogatorio médico puede suponer revictimización, una técnica de interrogatorio apropiada podrá minimizar sus efectos. ${ }^{5,14}$

El interrogatorio puede ser muy difícil de realizar, más aún si es un niño muy pequeño, poco colaborador, o con una actitud en la que se encuentra prácticamente paralizado por el miedo.

El interrogatorio y la valoración clínica general se deberán llevar a cabo previo consentimiento informado del paciente y encargados. La bibliografía especializada establece la conveniencia de interrogar al paciente por separado, para lo cual se deberá informar a los acompañantes del niño sobre la naturaleza de la entrevista, y ofrecerles la posibilidad de que observen a una distancia prudencial, mientras se interroga al menor; además, se le debe permitir salir del consultorio en el momento cuando lo desee, en busca de sus acompañantes. ${ }^{15}$

Es conveniente preparar el espacio físico de valoración para que sea lo más amistoso posible para los niños; de preferencia el médico se desprenderá de su gabacha, propiciará un periodo de empatía con el paciente y tratará de conocer aspectos generales de la vida del niño a través de técnicas diversas (dibujo, juego). Para conocer el ¿qué?, ¿cuándo?, ¿cómo? y ¿dónde?, se realizarán preguntas abiertas que no induzcan una respuesta determinada, por ejemplo: ¿Sabés por qué, o existe alguna razón para que te hayan traído a hablar conmigo?, etc. ${ }^{1,14,15}$

Una vez que el niño comienza a contar su relato, este se consignará exactamente en la forma como lo narra, transcribiendo en el expediente las mismas palabras que usó, sin juzgar o corregir su vocabulario. Si el menor no desea hablar, no se le presionará, y se podrá pasar a entrevistar a los acompañantes. ${ }^{14}$

\section{Lesionología general}

Debe sospecharse una lesión no accidental cuando un niño se presente con historia de algún traumatismo que no guarde relación con el mecanismo descrito en la historia médica suministrada, o bien, tenga traumatismos o cicatrices múltiples (criterios de Brown); ${ }^{6,7}$ la sospecha debe ampliarse si a través de la radiografía se diagnostican fracturas de distinta antigüedad, defectos en la consolidación, o fracturas que involucran metáfisis (criterios de Silverman). ${ }^{5}$

Los niños pueden ser agredidos en un hecho agudo o en varios durante un periodo larvado, pudiéndose observar contusiones simples o complejas de diferentes tiempos de evolución. El clínico debe ser cuidadoso en relación con establecimientos rígidos de tiempo de evolución de una lesión, puesto que existen diversos criterios médico legales, variaciones fisiológicas y fisiopatológicas que lo determinan. ${ }^{5-7,16}$
Entre el 81 y el $92 \%$ de los pacientes maltratados tienen lesiones de partes blandas, pero menos del 10\% de estas son de tipo "patrón". ${ }^{1}$ La equimosis patrón puede ser alargada (golpe con cables o cuerdas) o redondeada cuando se produce con un objeto de borde circular en un extremo (borde de un palo de escoba); ambas lesiones mostrarán el centro indemne. Algunas excoriaciones dan idea del objeto y mecanismo que las produce (rasguño, arañazo). Las equimosis o los hematomas profundos tardan en salir a la superficie, debido a los planos fasciales, por lo tanto, es posible una demora en su detección. ${ }^{1}$

Knight $^{16}$ apreció que una equimosis de tonalidad amarillenta debía tener no menos de 18 horas, presentándose una coloración rojiza, morada, azulada o negra desde una hora después de producida la lesión; además, equimosis derivadas de un mismo evento pueden resolverse en tiempos diferentes.

En el cuero cabelludo deberá buscarse la alopecia por arrancamiento ${ }^{5}$ el signo de la bandera en desnutrición. Las fracturas craneales simples y lineales generalmente se deben a caídas de menos de 4 pies de altura, y si bien pueden ser accidentales, deben considerarse sospechosas si son complejas (incluyendo las conminutas, diastásicas, desplazadas y las que cruzan de una línea de sutura a otra). ${ }^{1}$ Las petequias en rostro, mucosas e inclusive cuello, deben hacer pensar en un síndrome asfíctico (estrangulamiento, por ejemplo).

A nivel de la boca, se explorará el frenillo del labio superior, en busca de herida contusa que sugiere trauma tangencial, como en la bofetada, o un trauma directo; pueden verse excoriaciones en la mucosa bucal, por el intento de introducción de la cuchara en niños hipo o anoréxicos. ${ }^{4,5}$ No está de más la exploración de las piezas dentarias (luxación, fracturas). El trauma ótico por el golpe con manos abiertas contra los pabellones auriculares, puede producir la ruptura de la membrana timpánica. El síndrome del tirón de orejas causa contusiones en el pabellón auricular. ${ }^{4}$

En los miembros superiores suele observarse contusiones simples diversas y mordeduras, estas últimas deben medirse y describirse sus formas; preferiblemente tomar fotografía "uno a uno" y evitar el lavado para la toma de muestras de saliva. Usualmente las mordeduras humanas son poco profundas y dejan contusiones dispuestas en semiluna, por la impresión de los dientes. ${ }^{4}$ Puede solicitarse la valoración del odontólogo forense para comparación.

La quemadura en miembros inferiores, por sumersión infligida en agua caliente, respetará el área poplítea y la rodilla, ya que el menor al sentir el calor emanado del vapor, flexionará instintivamente los miembros; mientras que en la sumersión accidental, el menor no se percata y se introduce de pleno en el agua caliente, produciéndose una quemadura bastante simétrica de todo el miembro inferior hasta el nivel en que se encontraba el agua hirviente. ${ }^{1}$ La exposición 
durante un segundo de agua a una temperatura de $64^{\circ} \mathrm{C}$ es suficiente para producir una quemadura de segundo grado. ${ }^{17}$

En el abdomen las lesiones generalmente son observadas a nivel interno (laceración del hígado, bazo e intestino), por traumatismos directos. ${ }^{4}$ La mortalidad asociada a lesión visceral va del 40 al $50 \%{ }^{1}$ y puede verse fallo renal en casos de severas lesiones que produzcan rabdomiolisis y consecuente mioglobinuria. ${ }^{17}$

El trauma craneoencefálico es la causa más frecuente de muerte en menores. ${ }^{1}$ Un $95 \%$ de las lesiones intracraneanas severas y un $64 \%$ de todas las lesiones craneoencefálicas en menores de un año, son atribuidas a alguna forma de maltrato infantil. Además, el 80\% de las muertes por TCE en menores de dos años, se produce por lesiones no accidentales. Las fuerzas rotacionales severas pueden producir también daño axonal difuso. ${ }^{18}$

Aunque es frecuente que los niños sufran caídas, es muy poco común que presenten lesiones graves secundarias a esas. ${ }^{1}$ Puede ocurrir que niños por arriba de los cinco meses de vida (cuando son capaces de rodar sobre sí mismos e incorporarse) se precipiten de sus camas, no obstante, son extremadamente raras las lesiones graves por esto. Según un estudio que involucró a 782 niños menores de cinco años de edad, que habían sufrido precipitaciones accidentales desde una superficie elevada, como cama o sofá, 3 presentaron fracturas de clavícula; 6 , fracturas de cráneo; 1, fractura de húmero y 1, hematoma subdural. En otro estudio de 76 niños que se habían precipitado de una cama, cuna o silla de altura entre 1 y 3 pies, ingresados en un hospital por otras patologías, un $18 \%$ presentó hematomas faciales o craneales; un $12 \%$, laceraciones en la cabeza, y se vio una fractura de cráneo no desplazada y una fractura de hueso largo en paciente con osteogénesis imperfecta. ${ }^{1}$ Por último, datos obtenidos de estudios realizados por observadores de precipitaciones de las camas hospitalarias, literas y ventanas, han sido usados para estudiar la fuerza requerida para causar daño cerebral y fracturas en el niño, estableciéndose lo siguiente: una caída de 3 pies de altura puede raramente resultar en una fractura simple lineal del cráneo o clavícula; las caídas de una altura de 6 pies raramente producen conmoción cerebral, hemorragias subdurales o laceraciones.

Las fracturas transversales diafisiarias de huesos largos son producidas por fuerzas perpendiculares, y las espiroideas por fuerzas rotacionales. La fractura femoral es sugestiva de maltrato infantil en niños pequeños, puesto que en los mayores se pueden producir accidentalmente cuando se caen y sufren un mecanismo de torsión de la extremidad inferior. ${ }^{1}$ La fractura de manos y pies en niños mayores, generalmente obedece a accidentes, pero sugieren maltrato en niños pequeños. En un estudio de King y colaboradores, ${ }^{1}$ de 189 niños maltratados se encontró que el $60 \%$ estaban localizadas en el húmero, fémur y tibia, también hubo en el radio, cráneo, columna vertebral, costillas, cúbito y peroné (en orden decreciente de frecuencia). Puede haber fractura vertebral por compresión contra un objeto, generalmente secundaria a hiperflexión, pero esto es poco frecuente. ${ }^{1}$

El tiempo aproximado de evolución de las fracturas suele tener una cronología de formación perióstica, e inicio de callo óseo de entre 4 y 14 días; entre 10 y 21 días se ve callo óseo y la osificación se produce entre 4 y 8 semanas, con la línea de fractura aún visible. ${ }^{4}$

A nivel toxicológico puede demostrarse el uso de tranquilizantes para sedar al menor o hacerlo dormir, pero que además lo pueden intoxicar, o bien, ser emplusados sistemáticamente como en el Síndrome de Munchaussen by Proxy, en el que algún progenitor (generalmente la madre) crea enfermedades en los hijos, sometiéndolos a pruebas diagnósticas y terapéuticas innecesarias. ${ }^{1,4}$ El cuadro clínico muestra signos de la enfermedad simulados o inducidos por el agresor. ${ }^{1,17}$

\section{Síndrome del Niño Sacudido (SNS)}

En el SNS, ante un mecanismo de "zarandeo" en el que el menor es agitado de forma violenta en manos del agresor, la cabeza de gran tamaño proporcionalmente al tórax, se moviliza de adelante hacia atrás, produciendo un cuadro caracterizado por hematoma subdural (HS), como consecuencia del desgarro de los vasos "puente" entre el cráneo y el cerebro, asociado a edema, hemorragias retinianas (HR), fracturas costales $y$ en ocasiones fractura de

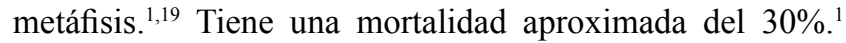
Puede asociarse a este mecanismo el impacto de la cabeza contra una superficie dura, y sus consecuentes lesiones craneoencefálicas. ${ }^{17}$ Este tipo de síndrome suele manifestarse en menores de dos años, ${ }^{4}$ pero se ha detectado en niños mayores. $^{18}$

El HS suele localizarse en la convexidad parietoccipital o en la cisura interhemisférica posterior. ${ }^{21}$ Las fuerzas que originan las lesiones craneoencefálicas exceden a las generadas por actividades normales de los niños, o por caídas de baja velocidad (movimiento en línea recta) que suelen producirse en el hogar ${ }^{17}$ además, debe tomarse en cuenta que lesiones del tipo caída son infrecuentes en menores que no caminan. Los hallazgos típicos obedecen a un evento violento y no pueden originarse en caídas cortas o durante convulsiones. ${ }^{21}$

El síndrome puede cursar con vómito, alteración del estado de conciencia, irritabilidad, dificultad para alimentarse, coma, convulsiones, dificultad respiratoria y estado de shock; también se ha observado la presencia de fiebre, fontanela prominente asociada a paresia $o$ hiperreflexia. ${ }^{1,17,20}$ Deben excluirse otras causas como: infección, intoxicación, trastornos metabólicos, etc. ${ }^{20,21}$ 
Entre el 75 y el $90 \%$ de casos de niños agredidos que involucraron una sacudida violenta, se produjo una HR difusa. ${ }^{18,21}$ Estas hemorragias suelen aparecer en las capas subretinal, intraretinal, preretinal, con o sin desdoblamiento de la retina en dos capas (retinosquisis). ${ }^{18,21}$ En la génesis se han postulado diferentes mecanismos de producción, como el aumento de presión venosa en la retina, por incremento de la presión intracraneal; la elevación de la presión intraocular debida a la compresión del tórax y la tracción de los vasos retinianos hacia el humor vítreo, a causa de las fuerzas de desaceleración angular. ${ }^{22}$ Se ha visto casos de HR por otras causas traumáticas; algunos ejemplos sobresalientes en la bibliografía son el caso de la precipitación de un bebé desde tres pies de altura, que produjo hemorragia epidural, fractura de cráneo y HR derecha; otro de cuatro meses, desde tres pies de altura, que produjo fractura de cráneo y hemorragia intraretinal izquierda (HIR), y otro desde cuatro pies de altura, que produjo hemorragia intracraneal, fractura de cráneo y HIR derecha. ${ }^{23}$

Otra causa de HR es la idiopática en recién nacidos; en un estudio se encontró que al analizar a 1238 neonatos, había una incidencia del $14 \%$ de HR al nacer, y del $2,5 \%$ entre el tercer y quinto día de nacimiento, por lo cual se supone que estas desaparecen con el tiempo. ${ }^{19}$ Las HR no son específicas para el diagnóstico, ni se puede establecer su data con precisión; se han reportado en algunos casos de accidentes de tránsito, ${ }^{21}$ especialmente asociadas a HS. Así es que las HR no son diagnósticas, pero sí sugestivas.

En casos en los que se asocia fracturas costales, se debe tener presente que se producen por la compresión anteroposterior de la caja torácica en manos del agresor, en la que el brazo de palanca que se produce en la costilla con la apófisis transversa, provoca la fractura del arco costal posterior (la localización usual es entre T4 y T9), aunque también pueden presentarse fracturas en el arco medio. ${ }^{1} \mathrm{Se}$ han observado fracturas costales antiguas, que pueden formar una imagen de lesión lítica expansiva similar a un tumor. ${ }^{1}$

En las maniobras de resucitación se ha visto que la elasticidad de la cavidad torácica infantil la hace capaz de soportar las compresiones, con fracturas costales solo en el $2 \%$ de los casos de una muestra de 94 niños estudiados. ${ }^{1}$

Una fractura muy sugestiva de maltrato infantil es la de la metáfisis en "asa de cubeta" o en "esquina metafisiaria", y que tienen su origen en los movimientos bruscos de estiramiento y torsión simultánea, provocados al tomar por las extremidades a los niños para balancearlos, golpearlos contra algún objeto o proyectarlos a distancia. ${ }^{24}$ Las fracturas metafisiarias son altamente sugestivas de abuso, puesto que las fuerzas necesarias para su producción no se explican por mecanismos de caída o accidentes simples. ${ }^{17}$

En general, fuerzas triviales, como aquellas relacionadas con juegos de rutina, columpiarse, o caídas de baja altura, son insuficientes para causar todo el cuadro del SNS. ${ }^{20}$

\section{Abuso sexual}

En Costa Rica, los delitos contra la libertad sexual se encuentran tipificados en el Código Penal (violación, abusos sexuales, etc.). ${ }^{11}$ El abuso sexual en niños consiste en la seducción y uso de un niño para que simule o lleve a cabo conductas de índole sexual, o sea sujeto pasivo de estas, y que se consigue por medio de la violencia, manipulación o intimidación. ${ }^{5,25}$ Se estima que el $25 \%$ de los niños víctimas de malos tratos o negligencia, han sido sometidos a abusos sexuales (mayor riesgo en las niñas que en los niños). ${ }^{1}$

En todo caso, deberán explorarse las áreas extra y paragenitales y la genital. ${ }^{6,7} \mathrm{El}$ examen de la niña se realizará con la paciente en posición "de rana", ginecológica, o sobre los regazos de su madre, con el método de separación o el de tracción de los labios mayores. Debe observarse la vulva completa, detallando las características del himen, la forma de su orificio (semilunar, anular, labiado, cribiforme, imperforado, etc.) y su diámetro. En adolescentes, para determinar si un himen es dilatable o dilatado, se introducen 1 y 2 dedos a través del orificio himenal, dependiendo de las características de distensibilidad del himen, observando si regresa o no a su estado original (dilatable y dilatado, respectivamente). ${ }^{26}$ Cuando el paso de un objeto, como lo puede ser el pene adulto erecto, distiende el himen más allá de su límite de elasticidad, produce una ruptura o desgarro de diversas características, dependiendo de la desproporción entre los genitales femeninos y el objeto utilizado. ${ }^{5}$ En todo caso, en las niñas menores de seis años el coito es anatómicamente imposible, pues el ángulo subpúbico es muy agudo, lo que constituye una barrera ósea; entre los seis y once años es posible, pero produce ruptura del periné o tabique rectovaginal, y entre los once años y la edad núbil, se puede producir la ruptura del himen. ${ }^{5}$

El desgarro ordinario llega al borde de inserción del himen, ${ }^{26}$ sin embargo, puede haber desgarros parciales, los cuales pueden cicatrizar formando una muesca, por lo que es necesario realizar un diagnóstico diferencial con las escotaduras congénitas, que pueden aparecer como parte de la anatomía de las niñas. ${ }^{5,27}$ Es preciso recalcar que los bordes del desgarro cicatrizan por separado, es decir, no sueldan jamás, por lo cual no se reconstituye la integridad de la membrana himenal, dejando lo que se ha llamado carúnculas mirtiformes. ${ }^{5}$ Algunos autores han descrito que la cicatrización clínica tiene lugar en un plazo de 3 a 4 días, ${ }^{5}$ sin embargo, un estudio histológico de Vargas Alvarado y Baudrit, indica que la cicatrización se produce a los diez días. ${ }^{26}$

El diámetro transverso del himen esperado para las niñas, va desde los $0,5 \mathrm{~cm}$ hasta $1 \mathrm{~cm}$ en las prepúberes, ${ }^{4}$ no obstante, las decisiones basadas en los diámetros himenales son riesgosas. De hecho, se describen grandes variaciones (incertidumbre estadística) en los diámetros himenales para cada edad, en pacientes menores que no han sufrido abuso sexual,${ }^{28}$ por lo cual, la regla que indicaba que por cada año se espera un milímetro de apertura himenal, está cuestionada. 
La controversia en cuanto a la relación entre el diámetro del orificio himenal y la relevancia con respecto a un supuesto abuso sexual, es mayor en tanto existen varias técnicas para explorarlo: separación frente a tracción de los labios, posición de la menor y el grado de relajación durante la exploración. ${ }^{29}$ Se conoce que el examen himenal no es un método infalible para confirmar o descartar el hecho delictivo (sobre todo en menores postpuberales) y, por otro lado, el daño genital no es la norma en los delitos sexuales..$^{30-32}$ Más aún cuando algunos estudios pequeños han sugerido que un himen traumatizado con una apertura grande puede regresar a su diámetro original en el curso de pocas semanas o meses, ${ }^{25}$ y por la información vertida en otro estudio retrospectivo con 36 adolescentes embarazadas, que encontró que solo 2 tenían hallazgos genitales "indicativos" de penetración vaginal. ${ }^{31}$

No se acepta que ejercicios violentos como la equitación, el baile, la separación brusca y amplia de las piernas, sean capaces de producir lesiones del himen, tampoco las maniobras masturbatorias (sin objetos) llevadas a cabo por la propia paciente, se aceptan como causantes de desgarro. ${ }^{5,29}$

Las adolescentes usuarias de tampón tienen menos daños himenales, probablemente porque el uso de tampones distiende el himen, haciendo que la penetración de un pene sea menor causa de lesión; sin embargo, esto también puede obedecer a que las que tienen orificios himenales más grandes o dilatables, se sienten más cómodas aplicándose el tampón. ${ }^{31}$

Debido al coito se puede producir otras lesiones, las cuales pueden involucrar el periné, tabique rectovaginal o fondo de saco vaginal. La fosa navicular y la posterior ("posterior fourchette") son asiento común de desgarros cuando se ha intentado forzar la penetración hacia el introito vaginal ("coito vulvar"). ${ }^{33}$

En varones puede observarse eritema o lesiones peneanas, como ruptura del frenillo o contusiones simples; en caso de que el menor haya sido sujeto pasivo de penetración por parte de una mujer, es necesario tomar muestras para determinar la presencia de células vaginales en los genitales del menor.

Las lesiones en el ano producidas por la penetración del pene erecto $\mathrm{u}$ objetos no son constantes, por lo que su objetivación tiene pocas posibilidades. En el atentado agudo pueden producirse excoriaciones, desgarros, fisuras, o no haber lesión alguna, sobre todo si de previo a la introducción del objeto se ha intentado la dilatación gradual del orificio anal. ${ }^{52}$ Para la evaluación del ano se recomienda examinar en la posición genopectoral, aunque se puede utilizar la posición mediolateral y completar el examen usando el tacto anal, para conocer el tono muscular de los esfinteres. También pueden observarse cicatrices, hipotonía y dilatación anales como parte del cuadro.
Conviene realizar el signo de la dilatación refleja del ano, de gran valor en casos de abuso sexual en niños en los que ha habido una dilatación anal forzada. Consiste en separar las nalgas del menor y observar. El esfinter externo puede contraerse voluntariamente durante 9 a 10 segundos (algunos autores indican que puede ser hasta durante 1 minuto, aproximadamente), ${ }^{27}$ por lo cual mantendrá el tono con el esfínter interno; un esfínter interno lesionado no podrá mantener el tono de este esfínter, de manera que se podrá observar directamente en la porción distal del recto. ${ }^{5}$

Ante un hallazgo anal deberá efectuarse de inmediato un diagnóstico diferencial, pues siempre habrá que tener presente la posibilidad de que obedezca a otras patologías.

\section{Toma de muestras}

La toma de las muestras se debe realizar utilizando guantes y evitando la contaminación con fluidos o elementos de transferencia propios del evaluador. Los aplicadores estériles por usar deben ser absorbentes y guardarse en paquetes secos y limpios; todas las áreas en las que haya habido contacto con fluidos biológicos deberán ser muestreadas. Para la toma de muestras en piel se aconseja la técnica del doble hisopo, descrita por Sweet, ${ }^{27}$ que consiste en usar agua estéril para mojar el aplicador, hacer rodar con poca presión el hisopo sobre el área de piel, y posteriormente aplicar un segundo hisopo seco para recolectar remanentes. Con el fin de localizar mejor el área contaminada, se sugiere el uso de la luz de Wood, y de preferencia la luz alternativa de Bluemaxx BM 500.27

Pueden recolectarse elementos pilosos o fibras encontrados en los genitales del menor, para tipificar y determinar si contienen fluidos. Es común la toma de residuos subungueales para estudiar por presencia de fluidos biológicos, células descamadas del agresor, etc.; para este efecto se tomarán los residuos usando un palillo con punta para cada dedo, y se empacarán por separado. ${ }^{27}$

Para determinar la presencia de semen, se pueden tomar muestras en boca, directamente con aplicadores estériles bajo la lengua, labios, paladar duro, o bien, obteniendo 10 $\mathrm{ml}$ de saliva, y luego tomar los aplicadores; posteriormente la boca se enjuaga con $10 \mathrm{ml}$ de agua destilada y se toma para análisis. Se considera que los espermatozoides pueden ser encontrados en cavidad oral durante 28 a 31 horas. Se ha visto que inclusive enjuagues bucales, tomar agua o lavarse los dientes, no necesariamente eliminan todas las trazas de espermatozoides. ${ }^{27}$

La cavidad vaginal se muestrea introduciendo el aplicador y rotándolo 360 grados; se prefiere el uso del espéculo, sin embargo, en menores es explicable su contraindicación. ${ }^{27}$ Se ha visto que los espermatozoides pueden encontrarse en vagina 8-24 horas después del abuso, pero se han encontrado inclusive entre tres y siete días, y en endocérvix, hasta 17 días. ${ }^{5,27}$ 
Algunos autores recomiendan la instilación con solución salina estéril (2-10 ml) en cavidad vaginal, y luego recoger con un gotero para obtener el material. 27,29

Las muestras anales se tomarán igualmente con aplicadores que se rotan; deben tomarse aún cuando el individuo haya defecado. ${ }^{27}$

Se deberán tomar muestras para enfermedades de transmisión sexual tan pronto como sea posible, y de HIV, de acuerdo con la normativa vigente.

El médico debe recordar que antes de considerar y proceder a la toma de muestras, es imprescindible asegurar la cadena de custodia de estas, pues de lo contrario pueden perder validez en los procesos judiciales. Además, las muestras deben conservarse en un medio de refrigeración y en paquetes adecuados, debidamente lacrados, con la boleta de cadena de custodia y las actas correspondientes.

Si no se cumple con lo anterior, el profesional deberá abstenerse de tomar las muestras y referir para la toma por parte del Departamento de Medicina Legal.

\section{Generalidades del diagnóstico}

Ante un menor agredido, la actuación del médico debe ser la de asegurar la supervivencia $\mathrm{y}$, secundariamente, realizar el examen médico legal. Se deberá efectuar una inspección de la actitud del menor, describir el estado de las ropas, explorar al niño con la menor cantidad de ropa posible y tomar las medidas antropométricas. ${ }^{4}$

En cuanto a exámenes de laboratorio, deberán incluirse: hemoleucograma, plaquetas, pruebas de coagulación, enzimas hepáticas y pancreáticas, si hubo traumatismo en el abdomen. ${ }^{1}$

Además de la valoración clínica completa, es necesario realizar estudios radiológicos y TAC, en ciertos casos. La Academia Americana de Pediatría indica que en todos los casos de sospecha de niño agredido menor de dos años, se exige un barrido óseo que incluye radiografía AP y lateral de cráneo, columna completa, tórax, extremidades (pies y manos) y pelvis; la British Society for Paediatric Radiology señala que el barrido debe efectuarse en los hermanos del paciente. ${ }^{110}$, 23, 34, 35 Según la Academia Americana de Cirujanos Ortopedas, no hay fractura patognomónica en el abuso. ${ }^{10}$ La resonancia magnética es útil para valorar lesiones intracraneales y sobre todo para dar seguimiento y control. ${ }^{4}$

Se recomienda repetir las radiografías algunos días después, para poner en evidencia hematomas subperiósticos iniciales ahora calcificados, y para determinar la existencia de hallazgos pasados por alto en el primer estudio.

Resulta muy importante estudiar los expedientes y notas médicas previas en los centros médicos en donde el menor haya sido atendido, para analizar los estados anteriores y los diagnósticos diferenciales. ${ }^{1}$

\section{Diagnóstico diferencial}

El diagnóstico diferencial es vasto y debe realizarse dependiendo de los hallazgos individuales que presenta el paciente.

\section{Pronóstico}

Deberá establecerse un seguimiento por parte de las instituciones que velan por la salud de los pacientes, como la CCSS y el PANI, ya que al retomar el hogar existen cifras que hablan de un $25 \%$ de remaltrato severo, y hasta un $15 \%$ de riesgo de ser asesinados. ${ }^{1}$

Las secuelas físicas dependerán del daño inicial provocado y la evolución del paciente; si bien pueden acaecer secuelas físicas, las psicológico-emocionales parecen ser las que producen más daño en el largo plazo. ${ }^{36}$

\section{Conclusiones}

El SMA es una patología social cuya intervención requiere un enfoque biopsicosocial. La valoración médica asistencial debe cuidar los aspectos médico legales al momento de interpretar la información clínica, ya que cualquier dato puede ser tomado en cuenta en los Tribunales de Justicia. Los profesionales deben conocer las aristas médico legales de esta patología, y actualizarse con respecto a la evidencia médica que cambia y aporta más información con el paso del tiempo.

\section{Referencias}

1. Beaty J, Kasser J, Rockwood, Ch. Fracturas en el niño. España: Marban, 2003: 242-264.

2. Loredo A. Maltrato físico y accidentes como causas de lesiones en el niño y utilidad de los indicadores clínicos para el diagnóstico diferencial. En: Maltrato en el niño. México: Mc Graw Hill Interamericana, 2001: 191-205.

3. Baeza C. Aspectos quirúrgicos del maltrato al menor. En: Maltrato en el niño. México: Mc Graw Hill Interamericana, 2001: 169-181.

4. María R. Violencia, Salud infantojuvenil y calidad de vida. En: Maltrato en el niño. Mc Graw Hill Interamericana, 2001: 43-62.

5. Calabuig G. Medicina legal y toxicología. Quinta ed. España: Masson. 1999: 445-448.

6. Vargas E. Medicina legal. México: Trillas, 1998: 257-259.

7. Vargas E. Medicina forense y deontología médica. México: Trillas, 1991: 632-640

8. OMS. Report of the consultation child abuse prevention. Ginebra: WHO, 1999: 29-31.

9. Castellanos J. La medicina legal preventiva, actuación del médico legista ante la violencia familiar contra el menor. En: Maltrato en el niño. México: Mc Graw Hill Interamericana, 2001: 83-97.

10. Colleen K. Child abuse: approach and management. Am Fam Physician 2007; 75: 221-228.

11. Zuñiga U. Código Penal. Costa Rica: IJSA, 2002: 82, 87-91, 74-76, 182-183. 
12. Asamblea Legislativa de Costa Rica. Código de la Niñez y la Adolescencia. Costa Rica: Publicaciones Jurídicas. 2002: 14, 37.

13. Marcón O. Tras el abuso, revictimización. El Santafesino. 2005. En: $\quad$ http://www.elsantafesino.com/opinión/2005/03/18/3420. Consultado el 15 de junio de 2006

14. Madrigal E, Roldán J. La historia médico legal en casos de delitos sexuales en niños. Med Leg Costa Rica. 2007; 24: 107-122.

15. Cantón J, Cortés M. Guía para la evaluación del abuso sexual infantil. Barcelona: Pirámide, 2000.

16. Knight B. Forensic Pathology. London: Arnold, 1996: 139-145.

17. Fernández G, Perea J. Síndrome del maltrato infantil. Rev Cubana Pediatr 2004; 76: 3 .

18. Shalehi-Had H, Brand J, Rosas A, et al. Findings in older children with abusive head injury: Dos shaken child syndrome exist? Pediatrics 2006; 117: 1039-1044.

19. Caffey J. The whiplash shaken infant syndrome: Manual shaking by the extremities with whiplash induced intracraneal and intraocular bleedings, linked with residual permanent brain demage and mental retardation. Pediatrics 1974; 54:4.

20. Duhaime A, Christian C, Balian L. Nonaccidental head injury in infants "The shaken baby syndrome". N Engl J Med 1998; 338: 1822-1829.

21. American Academy of Pediatrics. Shaken Baby Syndrome: rotational cranial injuries techical report, Committee on Child Abuse and Neglect. Pediatrics 2001; 108: 206-210.

22. Yamin G. Síndrome del niño sacudido. En: Maltrato en el niño. Mc Graw Hill Interamericana, 2001: 206-217.

23. Bechtel K, Stoessel K, Leventhal J. Retinal Hemorrhages in Accidental and Nonaccidental Injury: In Reply. Pediatrics 2005; 115: 192.
24. Gómez I, Serrano I, Martínez M. Imaging diagnosis of non accidental injury in children. Cuad Med Forense 2006; 12: 21-29.

25. Wissow L. Child Abuse and Neglect. N Engl J Med 1995; 332: 1425-1431.

26. Roldán J. Himen. Med Leg Costa Rica. 1986; 3:2-4.

27. Stark M. Clinical Forensic Medicine: A Physicians guide. England: Humana Press 2005: 61-127

28. McCann J, Wells R, Simon. Genital Findings in prepubertal girls selected for nonabuse: a descriptive study. Pediatrics 1990; 86: 428439.

29. Giardino A. A Practical guide to the evaluation of sexual abuse in the prepubertal child. U.S.A.: Sage Publications. 1992: 29-81

30. Underhill R, Dewhurst, J. The doctor cannot always tell. Medical examination of the "intact" hymen. Lancet 1978; 311: 375-376.

31. White C, McLean I. Adolescent complainants of sexual assault; injury paterns in virgin and no virgin groups. Journal of Clinical Forensic Medicine 2006; 13: 172-180.

32. Wells D. Sexual assault practice: myths and mistakes. Journal of Clinical Forensic Medicine 2006; 13: 189-193.

33. Girardin B, Faugno D, Seneski P. Coloratlas of sexual assault. EUA: Mosby, 1997:25-26

34. Day F, Clegg S, McPhillips M. A retrospective case series of skeletal surveys in children with suspected non accidental injury. Journal of Clinical Forensic Medicine 2006; 13: 55-59.

35. Porras R, Pérez V. Síndrome del niño sacudido: la importancia de las hemorragias retinianas y hematomas subdurales en su diagnóstico. Acta pediátr costarr 2002; 16: 2 .

36. Bass E, Davis L. El coraje de sanar. Barcelona: Urano, 1994. 